Communications in Physics, Vol.21, No. 3 (2011), pp. 199-209

\title{
ON THE ROLE OF MAGNETIC FIELD FLUCTUATIONS IN DIFFUSIVE SHOCK ACCELERATION
}

\author{
DOAN KIM THE, PHAM TUAN ANH, PHAM NGOC DIEP, PHAM NGOC DONG, \\ NGUYEN VAN HIEP, DO THI HOAI, PHAM THI TUYET NHUNG, NGUYEN THI \\ THAO AND PIERRE DARRIULAT \\ VATLY, Institute for Nuclear Science and Technology
}

\begin{abstract}
A simple numerical simulation of the mechanism of diffusive shock acceleration, responsible for the acceleration of cosmic rays in the environment of young Super Nova Remnants, is presented. The relative roles of a uniform magnetic field component, inherited from the parent collapsed star, and of magnetic turbulences, known to be present in the vicinity of the shock, are investigated. It is shown that a uniform magnetic field allows for only doubling the energy of the cosmic particle. Important accelerations require the simultaneous presence of magnetic field turbulences at a scale commensurable with its Larmor radius.
\end{abstract}

\section{INTRODUCTION}

The sources of galactic cosmic rays, which form the bulk of those that reach the Earth, have long been unknown. It is only recently, with the advent of X-ray astronomy in space and of gamma-ray astronomy on ground, that it became possible to identify young Super Nova Remnants (SNR) as the most common sources.

Cosmic rays are atomic nuclei, their electric charge implies important distortion of their trajectories in the disc of the Galaxy and, as a result, it is not possible to tell where they come from. Only at the very highest energies, well above the EeV scale, is it possible to associate cosmic ray showers with optical, X-ray or radio counterparts in the sky [1]. Contrary to cosmic rays, gamma rays travel straight in the universe and point back to their sources. They are good at revealing the high energy decay photons coming from neutral pions produced in the interaction of very high energy cosmic rays with interstellar matter (ISM). Gamma ray astronomy detects high energy gamma rays from the Cherenkov light produced by electrons and positrons in the showers induced by their interaction with the upper atmosphere. Such showers are similar to the extensive air showers induced by cosmic rays, but significantly shorter because the radiation length is significantly shorter than the interaction length. Main sources of high energy photons are free-free scattering (bremsstrahlung) and synchrotron radiation at low energies and $\pi^{0}$ decays or inverse Compton on the cosmic microwave background at higher energies. Accurate observations in the $\mathrm{TeV}$ range have been made by the High Energy Stereoscopic System (HESS) in Namibia. It includes four telescopes [2] at the corners of a $120 \times 120 \mathrm{~m}^{2}$ square, operating above $100 \mathrm{GeV}$. Its field of view is $5^{\circ}$ and its resolution a few arc minutes. HESS has shown that many sources have an X-ray counterpart identified as SNR. In 2004, 
in the wake of earlier less accurate observations [3], HESS made a $33 \mathrm{~h}$ live time observation [4] of SNR RX J1713, which, when compared with an X-ray image taken by Rosat in 1996 [5] established that the SNR shell [6] is the source of the gamma ray signal. Later on, higher resolution X-ray observations [7] revealed the presence of important turbulences in the region of the shock with important variations from one year to the next. Many such observations followed, with improved resolution, establishing this way that galactic cosmic rays originate from SNRs.

The identification of SNRs as sources of galactic cosmic rays has suggested an acceleration mechanism, called diffusive shock acceleration (DSA), which is now accepted as the most likely candidate for accelerating cosmic rays [8]. As in a cyclotron, the particle is accelerated locally on traversing the shock (equivalent of the gap between the cyclotron dees) and is guided by magnetic fields on either side in such a way as to come back to the shock (equivalent of the dipole guide field of the cyclotron). However both the acceleration and guiding processes are very different from the cyclotron case. Guiding is provided by the remnant magnetic field inherited from the parent star, now strongly diluted, and by stochastic collisionless scattering on magnetic turbulences. Indeed, the mean free path is so large in the much diluted ISM that collisions can be neglected and the two colliding media can be seen as carrying nothing else than magnetic fields.

Acceleration is best described in the frame where the particle happens to be and where the magnetic fields are at rest: while bending the particle trajectories, they do not affect its energy, which therefore remains constant. Essential to this argument is the very low density of the ISM in which the particle evolves, making the occurrence of collisions negligible $^{1}$. Eventually, the particle returns to the shock and crosses it. The Lorentz transformation that describes the movement on the other side of the shock always implies acceleration as both media aim at each other with relative velocity $\beta$. At each shock traversal, the energy $E$ of the particle increases by $\Delta E$ such that $\Delta E / E=\beta$. After $n$ shock traversals it reaches $E_{n}=E_{0}(1+\beta)^{n}$. At some point, however, the particle drifts too far from the shock to have a chance to return to it and escapes.

The aim of the present article is to simulate this acceleration mechanism and to evaluate the role played by magnetic turbulences.

\section{A SIMPLE SIMULATION: GENERAL FRAMEWORK}

In order to become familiar with the process of diffusive shock acceleration and to understand its main features, we designed a simple numerical experiment. The shock is taken to be planar and progresses at velocity $\beta_{\text {shock }}$ in the ISM (in units of light velocity). Typically, we are dealing with an SNR having expanded at $1 \%$ of light velocity for $1 \mathrm{kyr}$, meaning a radius of some 10 light years (which justifies the planar approximation), some $10 \%$ of the final radius before entering the final Sedov phase and dissolving into the ISM. As magnetic field amplification near the shell of young SNRs produces fields of several hundred $\mu G$ [9], we shall deal with magnetic fields of up to $1 \mathrm{mG}$, namely up to $10^{-7} \mathrm{~T}$.

\footnotetext{
${ }^{1}$ For an ISM density of $1 / \mathrm{cm}^{3}$ and a cross-section of 1 barn $\left(10^{-24} \mathrm{~cm}^{2}\right)$ the mean free path of a relativistic proton is indeed $10^{6}$ light years!
} 
Over a distance of $10^{9} \mathrm{~m}$, or $\sim 3$ light seconds, the transverse momentum kick given to a proton is therefore up to $\sim 30 \mathrm{GeV} / \mathrm{c}$.

The $x$ axis is taken normal to the shock pointing upstream (meaning toward the undisturbed ISM). Space is divided in cells of equal sizes. Units of length are light seconds, of momentum $\mathrm{GeV} / \mathrm{c}$, of energy $\mathrm{GeV}$ and of magnetic field $\mu \mathrm{G}$. One unit of field over one unit of distance means a transverse momentum kick of $9 \mathrm{MeV} /$ c, i.e. 0.009 units of energy. For a field $H[\mu G]$ over a distance $l$ [light seconds], a proton of momentum $\boldsymbol{p}[\mathrm{GeV} / \mathrm{c}]$ gets a transverse momentum kick of $\Delta \boldsymbol{p}=0.009 \boldsymbol{H}_{\wedge} \boldsymbol{l}$. Its Larmor radius is $R=111 \mathrm{p} / \mathrm{H}$. Cell sides are accordingly chosen to be one light second. Field irregularities of the same scale are generated by locating a magnetic dipole in the centre of each cell.

Particles (assumed to be protons) are tracked using a Runge-Kutta algorithm with a step of 0.03 Larmor radii, that is $3.33 p / H$, but never exceeding 0.1 light second. For relativistic particles, to increase $p$ and $H$ by a same factor leaves the Larmor radius invariant and, for a same magnetic field map, leaves the particle trajectories invariant. Conversely, to leave $p$ invariant but to increase the magnetic field amplitude by a same factor everywhere decreases the Larmor radius by that factor and has therefore the same effect as to expand the spatial scale of the magnetic field map by that same factor. This basic scaling property of the problem is made use of to simulate field configurations having different values of the distance $D$ between neighbour dipoles. The movement of the shock front obeys the same scaling law and does not need to be modified.

Calculations are performed in the frame of the medium in which the particle happens to be. This means that a Lorentz transformation is made at each shock traversal. Upstream, one sees the shock approach at a velocity $\beta_{\text {shock }}$ and downstream one sees the shock moving away at a velocity of $0.25 \beta_{\text {shock }}$ under the assumption that the compression ratio is 4 , as obtained for monatomic gases in hydrodynamics. In each frame, upstream or downstream, one sees the other medium approach at a velocity $0.75 \beta_{\text {shock }}$.

Space is divided in cubic cells of sides equal to 1 light seconds. The magnetic field is given in $100 \times 50 \times 50$ cells surrounding the origin. In each cell one gives the value of a mean field (constant over the cell) and that of the magnetic moment of a magnetic dipole located at the centre of the cell. In any given point, the magnetic field is calculated as the sum of the mean field in the cell containing the point and of the twenty-seven dipole fields of the twenty-seven cells surrounding the point.

We recall that the field at a vector distance $\boldsymbol{r}$ from a dipole magnetic moment $\boldsymbol{M}$ is, up to a constant factor, $\boldsymbol{H}=\left(3 \boldsymbol{r}(\boldsymbol{M} . \boldsymbol{r})-\boldsymbol{M} r^{2}\right) / r^{5}$. At unit distance along the magnetic moment, $\boldsymbol{H}$ is directed along the magnetic moment and equal to $2 \boldsymbol{M}$. At unit distance in the equatorial plane, $\boldsymbol{H}$ is directed against the magnetic moment and equal to $-\boldsymbol{M}$. A dipole field is the field generated by a current loop in the approximation where the loop radius can be neglected. Indeed, $\boldsymbol{H}$ as given above diverges at $r=0$. In the case of a current loop, the real field differs from the dipole field at distances to the centre at the scale of the loop radius. In order to obtain a realistic field shape, it is therefore necessary to replace the dipole field by another expression when approaching the centre. In the present simulation, the field is taken to be the dipole field outside and on a sphere of radius 0.5 . Inside the sphere, the field is linearly interpolated between the value on the sphere at the intersection with the line joining the point to the centre and the central 

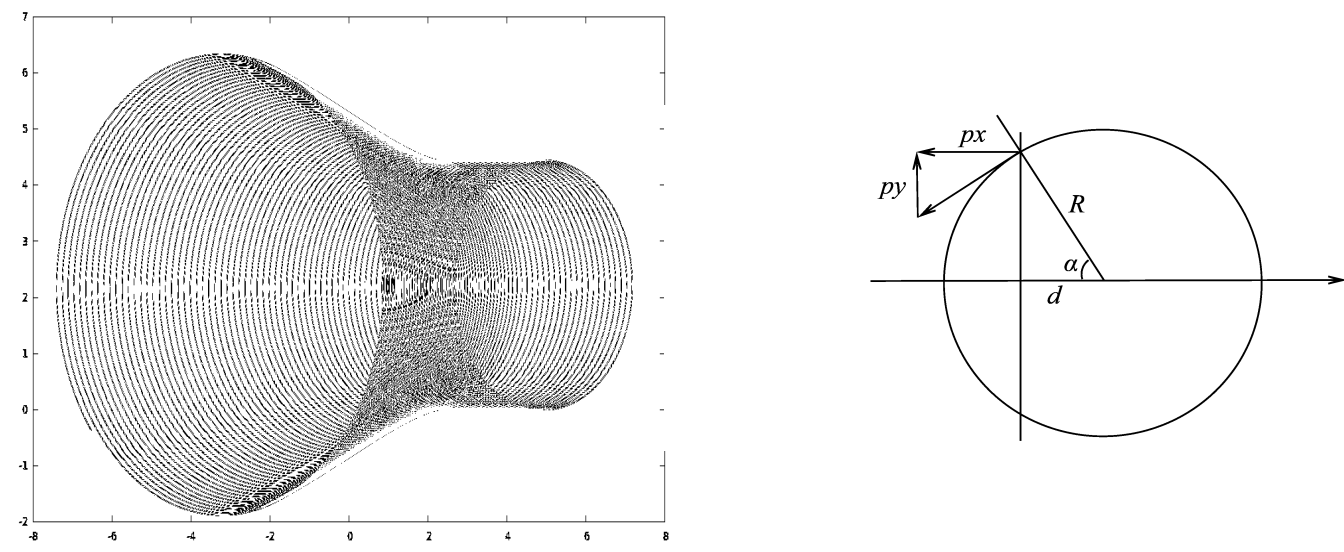

Fig. 1. Left: Trajectory of a particle accelerated in a uniform magnetic field. Note that what is plotted is not in real space but in the rest frame of where the particle happens to be. Namely the shock stays at $x=0$ and both the upstream and downstream trajectories are drawn in half-spaces that are at rest. Right: Schematic geometry of the uniform magnetic field case.

value obtained with a current loop of magnetic moment $\boldsymbol{M}$. A current loop of radius $R$ and current $I$ has $M=\pi I R^{2}$ pointing along the loop axis and the field in the centre is, up to the same constant factor as above, $2 \pi I / R=2 M / R^{3}$. Taking the loop around the sphere equator gives a field equal to $16 \mathrm{M}$ in the centre of the loop. The field on the sphere is $8(3 \hat{\boldsymbol{r}}(\boldsymbol{M} . \hat{\boldsymbol{r}})-\boldsymbol{M})$ where $\hat{\boldsymbol{r}}$ is the unit vector along $\boldsymbol{r}$. The interpolation goes therefore between $16 \boldsymbol{M}$ and $-8 \boldsymbol{M}$ in the equatorial plane; along the dipole axis, the field stays constant and equal to $16 \boldsymbol{M}$.

\section{UNIFORM MAGNETIC FIELD}

In order to get some familiarity with the main features of the process, it is useful to start looking at the simple case of a uniform magnetic field. As it is the components of the magnetic field parallel to the shock that cause shock traversals, it is sufficient to consider a magnetic field $H$ directed along $z$. Proton trajectories are helices projecting as circles in the $(x, y)$ plane. There is no loss of generality in assuming that the initial proton momentum is in the $(x, y)$ plane, implying circular trajectories. Fig. 1 (left) shows the trajectory of a relativistic proton starting upstream with a momentum $p_{0}\left(\sim E_{0}\right)=10 \mathrm{GeV}$ in a field $H=500 \mu \mathrm{G}$. Its radius is therefore $R_{0}=2.2 \mathrm{light}$ seconds. It takes $2 \pi R_{0}=13.8$ light seconds for the proton to go around. The trajectory displayed in Fig. 1 is for a shock velocity $\beta_{\text {shock }}=0.01$. When the proton has made a turn, the shock has therefore approached by 0.138 light seconds. In the initial phase, the proton energy remains constant and equal to $10 \mathrm{GeV}$ until its trajectory reaches the shock.

After completion of this initial phase of approach, the trajectory crosses the shock, twice per turn. We note $d=-R \cos \alpha$ the distance from the centre of the circle to the shock (Fig. 1 right), $\alpha$ varies from 0 to $\pi$ and $d$ from $-R$ to $R$ while the shock scans through 
the circle. The relative energy gain per turn is proportional to the $x$ component of the momentum, namely $\Delta E / E=2 \sin \alpha \times 0.75 \beta_{\text {shock }}$. The radius of the trajectory accordingly increases by the same relative amount at each turn, $\Delta R / R=\Delta E / E=1.5 \sin \alpha \beta_{\text {shock }}$. At crossing, as $d / R=p_{y} / p=\cos \alpha, d=p_{y} R / p=$ cte. Indeed, $p_{y}$ is invariant and so is the ratio $R / p$. At each new crossing, $R, p$ and $p_{x}$ all grow but $d$ remains constant. Hence

$\Delta d=0=-\Delta R \cos \alpha+R \sin \alpha \Delta \alpha$ and $\Delta \alpha=1.5 \cos \alpha \beta_{\text {shock}}$. The angle $\alpha$ first grows when the shock is downstream the centre of the circle and then decreases when the shock is upstream. After crossing, $R$ stays constant but $d$ starts increasing: the particle spends a time $2 R \alpha$ in the downstream region or $2 R(\pi-\alpha)$ in the upstream region; each full turn, the shock advances by $\Delta d=2 R \beta_{\text {shock }}(0.25 \alpha+\pi-\alpha)=2 R \beta_{\text {shock }}(\pi-0.75 \alpha)$ with respect to the particle trajectory. As $R$ is now constant,

$\Delta d=R \sin \alpha \Delta \alpha$ and $\Delta \alpha=\Delta d /(R \sin \alpha)=2 \beta_{\text {shock }}(\pi-0.75 \alpha) / \sin \alpha$.

Therefore, at each turn, $\alpha$ increases by $1.5 \cos \alpha \beta_{\text {shock }}$ when crossing the shock and by $2 \beta_{\text {shock }}(\pi-0.75 \alpha) / \sin \alpha$ when circling around. Hence

$\Delta \alpha / \beta_{\text {shock }}=1.5 \cos \alpha+2(\pi-0.75 \alpha) / \sin \alpha$

$\Delta E / E=1.5 \sin \alpha \Delta \alpha /\{1.5 \cos \alpha+2(\pi-0.75 \alpha) / \sin \alpha\}$

Writing $F(\alpha)=\int 1.5 \sin x d x /\{1.5 \cos x+2(\pi-0.75 x) / \sin x\}$ where the integral runs from 0 to $\alpha$, the particle energy is $E=E_{0} \exp (F(\alpha))$. When the shock has scanned through the trajectory, the particle energy has increased by a universal factor ${ }^{2} \exp (F(\pi))=2$ : as long as the first trajectory is upstream and does not cross the shock, it will always ultimately drift fully downstream and the energy will have doubled independently from the values of the magnetic field, of the shock velocity and of the initial momentum.

After the acceleration phase, the proton stays on its fixed circular orbit in the downstream region while the shock keeps drifting away. A uniform magnetic field is therefore unable to produce significant acceleration.

\section{ADDING RANDOM DIPOLES}

We now show that space fluctuations in the magnetic field allow for reaching larger accelerations than with a uniform field by considering a magnetic field made of a superposition of a uniform field $H_{0}$ along the $z$ axis and of dipoles having a common magnetic moment $M_{0}$ and random orientations. By changing the values of $H_{0}$ and $M_{0}$ one learns about their relative roles. It was soon realized that an important element of the simulation was the criterion used to decide when the particle has escaped with no chance to return to the front region. We have been attentive to make sure, in each single case, that a safe criterion was being used. Similarly, attention has been paid to make sure that the results reported here are not significantly affected by the choice of initial position of the particle.

Figure 2 shows typical trajectories, projected on the $(x, z)$ plane, of a $10 \mathrm{GeV} / \mathrm{c}$ proton having a Larmor radius of order unity in the constant field $\left(H_{0}=1 \mathrm{mG}\right)$. They illustrate how $M_{0}$ should not be too small in comparison with $H_{0}$ in order to efficiently confine the particle in the vicinity of the shock. We have seen in the preceding section that

\footnotetext{
${ }^{2}$ Indeed, the integrand reads $d u / v$ with $v=0.75(2 \cos x \sin x-2 x)+2 \pi=0.75(\sin 2 x-2 x)+2 \pi$ and $d u=1.5 \sin ^{2} x=0.75(1-\cos 2 x)$. Hence $d u=-d v / 2$ and $F(\alpha)=\left\{(\log v)_{\alpha=0}-(\log v)_{\alpha=\pi}\right\} / 2$. Hence $E / E_{0}=\exp (F[\pi])=\sqrt{\left\{(v)_{\alpha=0} /(v)_{\alpha=\pi}\right\}}=2 \pi /(\pi / 2)=\sqrt{4}=2$.
} 


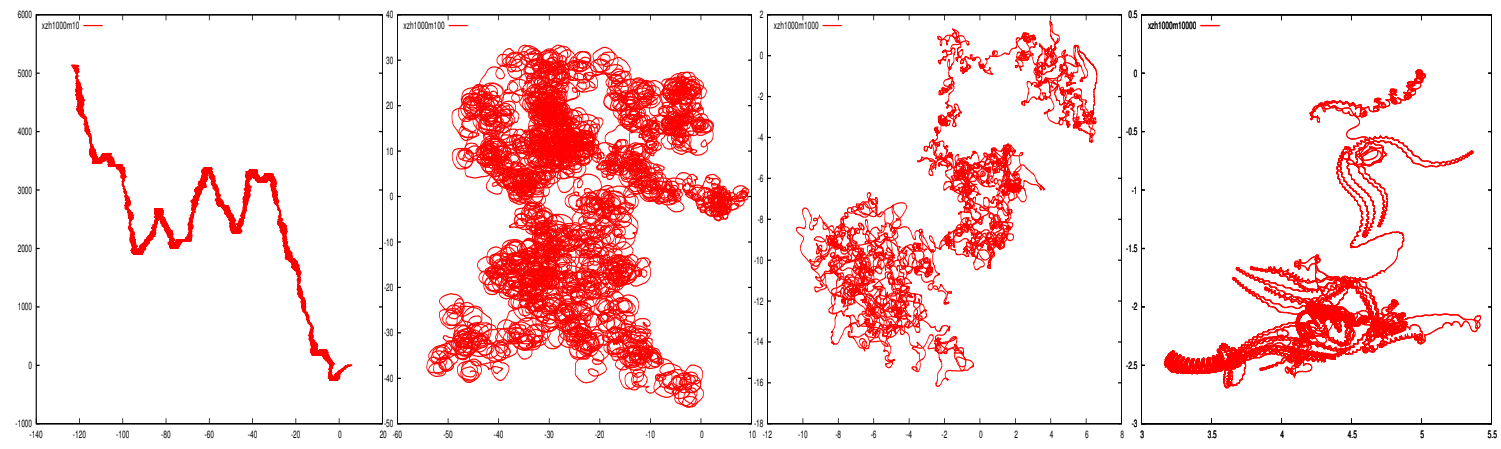

Fig. 2. Trajectories in the $(x, z)$ plane of a $10 \mathrm{GeV}$ proton in a field of $1 m G$ with added fluctuations such that $M_{0} / H_{0}=1 \%, 10 \%, 1$ and 10 (from left to right).

in the absence of fluctuations the trajectories remain in the $(x, y)$ plane and keep drifting away from the front. For $M_{0} / H_{0}=1 \%$ (left panel) the fluctuations are still insufficient to bring the particle back to the shock: it drifts away downstream. Moreover, occasional diffusions on a dipole making a large angle with the $z$ axis transfer momentum away from the $(x, y)$ plane to the $z$ direction and the particle may then keep drifting along the $z$ axis for a long time. For $M_{0} / H_{0}=100$ (right panel) the Larmor radius associated with the fluctuations is much smaller than that of the uniform field: the particle trajectory is locally a helix which displays characteristic magnetic mirror features, the particle being reflected when the pitch angle reaches $90^{\circ}$, as happens near the poles of the earth in the case of auroras. In between, for the central panels, and in particular for $M_{0} / H_{0}=10 \%$ (central left panel) a good confinement is achieved and accelerations of two to three orders of magnitude can be obtained.

The ratio between $M_{0}$ and $H_{0}$ is not the only relevant parameter. The amplitude of the field is equally important and the acceleration is more and more efficient as it increases. However, it is not sensible to increase the magnetic fields beyond reason, say above $1 \mathrm{mG}$ or so. What can be done instead is to change the distance between neighbour dipoles. Indeed, one obtains identical trajectories and energy spectra when the magnetic fields are multiplied by a constant $k$ and the distances divided by the same constant $k$. The scaling law that governs trajectories and energy spectra also applies to the drift of the shock front that proceeds at constant velocity as does the particle (assumed to be relativistic) on its trajectory. For an efficient acceleration, one may therefore expect that both $H_{0}$ and $M_{0}$ on the one hand, and the distance $D$ between neighbour dipoles and the Larmor radius $R$ on the other, must obey some relation.

\section{ENERGY SPECTRA}

Energy spectra have been calculated for different values of $M_{0}$ and $H_{0}$. The distance $D$ between neighbour dipoles was kept equal to 1 light second and the fields were allowed to take values well outside what has been considered reasonable. The results of the present 
section must therefore be understood having in mind the scaling law described in the preceding section: the trajectories and energy spectra obtained with a $100 \mathrm{mG}$ field must be interpreted as applying to, say, a $100 \mu G$ field and a distance $D$ between neighbour dipoles equal to 1000 light seconds. The initial momentum is taken equal to $10 \mathrm{GeV}$ and the shock velocity equal to $2 \%$ of the light velocity. The initial position of the particle is chosen at random in the upstream region. The efficiency of the acceleration, from initial energy $E_{0}$ to final energy $E$, is measured by a parameter $\rho=\left\langle\log _{10}\left(E / E_{0}\right)\right\rangle$ and the index $n$ obtained from the best fit of the final energy distribution to a power law. Fig. 3 shows the dependence of $\rho$ on $M_{0}$ and $H_{0}$ for $D=1$ light second. It gives evidence for a favoured relation between $H_{0}$ and $M_{0}$ with $M_{0} / H_{0}$ increasing from $\sim 0.2$ to $\sim 0.5$ when $H_{0}$ increases from 10 to 10000 . This relation corresponds to a kind of resonance between the respective roles of $H_{0}$ and $M_{0}$. If the fluctuations are too small they cannot sufficiently modify the circular trajectories and prevent them from drifting away downstream. In the absence of a uniform field component, the particle follows a random walk trajectory and takes much longer to return to the front than in the case of a uniform field where a single circle is enough. During that time, the front moves farther away than in the uniform field case. The optimum is a compromise where the fluctuations are sufficiently larger than the uniform field to prevent the particle from drifting away and where the uniform field is sufficiently large for its coherent action to help bringing the particle back to the front more efficiently than a random walk would.

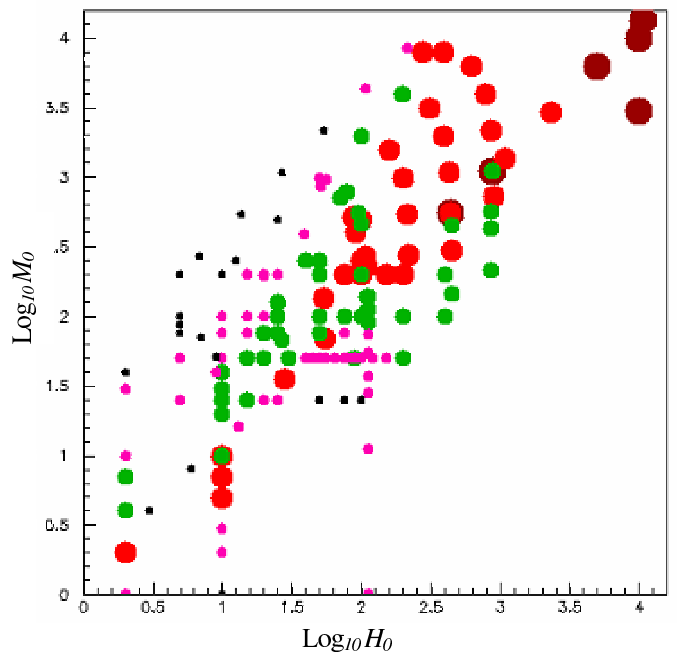

Fig. 3. Distribution of $\rho$ in the $\left(\log _{10} H_{0}, \log _{10} M_{0}\right)$ plane. The size of the dots increases with the value of $\rho$ in the following intervals: $<0.3,0.3$ to $0.35,0.35$ to $0.4,0.4$ to 0.425 and $>0.425$
When such acceleration conditions are satisfied, the acceleration becomes more and more efficient when the amplitude of the field increases, namely when the ratio between $D$ and $R$ also increases. The acceleration efficiency is found to display a broad maximum corresponding to an average Larmor radius of typically a percent of the distance between neighbour dipoles. In such a regime, the magnetic mirror effect is very strong and the predictions of the simulation become significantly model dependent. What happens in practice is that the particle is trapped between two neighbour dipoles during a long period of time. If the particle is injected far away from the front it never reaches the front. If it is injected close enough to the front, as is done in the simulation, it may get trapped between two neighbour dipoles on either side of the front. It is clear that the idealization of the structure of perturbations made in the simulation becomes inadequate in such 
a case. In reality, $\rho$ reaches its maximum (between 0.6 and 0.7 ) for Larmor radii of the order a percent of the distance between neighbour dipoles and higher fields, or equivalently smaller Larmor radii, are dominated by magnetic trapping and cannot be reliably modelled without having a precise knowledge of the real perturbations. Fig. 4 illustrates this well. It displays energy spectra obtained for the following values of the magnetic field: $H_{0}=M_{0}=10000,30000$ and 100000. In the case of the lower field value, with Larmor radii of the order of $10 \%$ of the distance between neighbour dipoles, the energy spectrum is still a power law with index close to unity, as it is for lower field values whenever $H_{0}$ and $M_{0}$ are in the resonance region. But when the fields increase to 30 and $100 \mathrm{mG}$, namely when the Larmor radii reach the percent level and below, a bump appears at large accelerations, associated with particles being trapped between two dipoles on either side of the front.
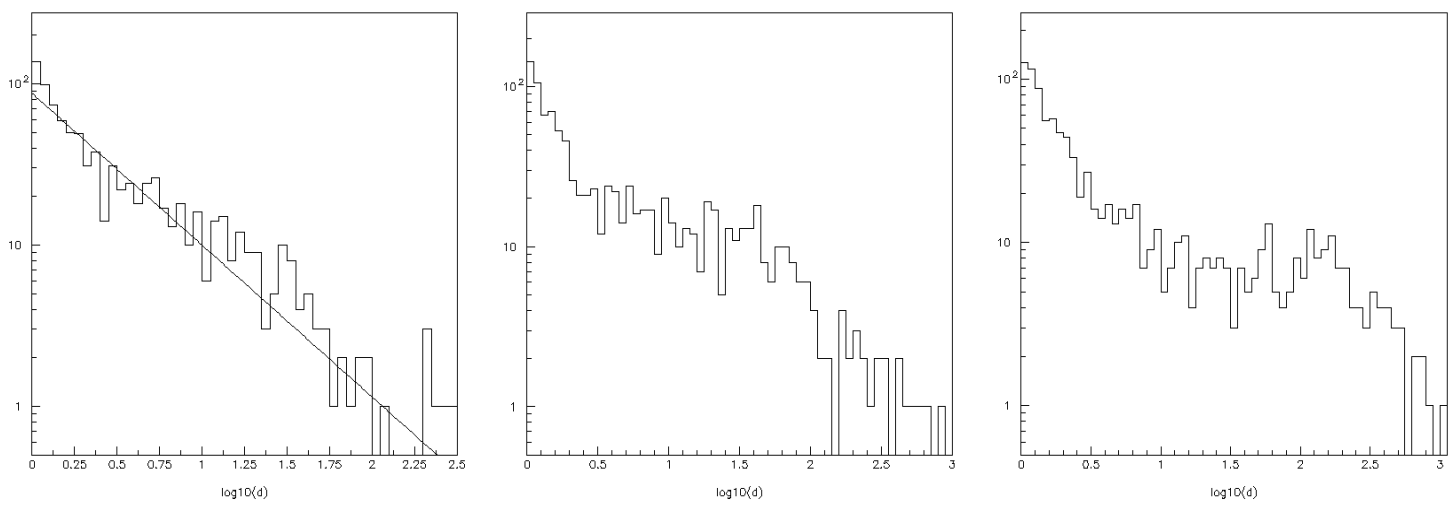

Fig. 4. Distribution of $\log _{10}\left(E / E_{0}\right)$ in the $H_{0}-M_{0}$ resonance region when moving toward smaller $R / D$ values. $H_{0}=M_{0}=10000,30000,100000$ and $\rho=0.46,0.61$, 0.65 from left to right respectively. The higher $R / D$ case is fit to a power law with index $\sim$ unity (black line). When $R / D$ decreases a bump appears at large accelerations, associated with magnetic trapping between neighbour dipoles.

In summary, two conditions must be fulfilled for the acceleration to be efficient: $H_{0}$ and $M_{0}$ must obey a resonance relation and the Larmor radii must be small enough, say below a few percent, in comparison with the distance between neighbour dipoles. The resonance condition between $H_{0}$ and $M_{0}$ implies that their ratio be between $\sim 20 \%$ at low fields and unity at high fields. Otherwise, the energy spectra are cut-off because the particle escapes prematurely the shock region (Fig. 5).

\section{SUMMARY AND CONCLUSIONS}

The simple simulation of the mechanism of diffusive shock acceleration presented here has illustrated several of its most important features. The main result has been to 


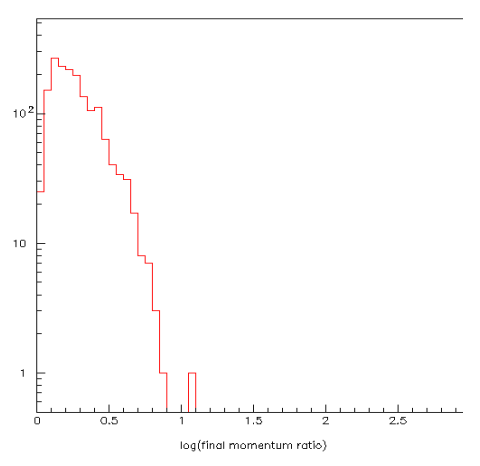

(a)

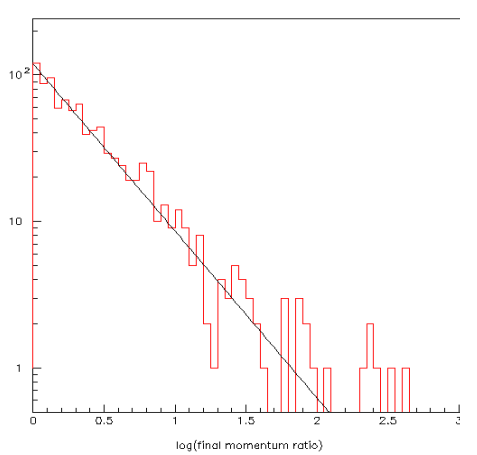

(b)

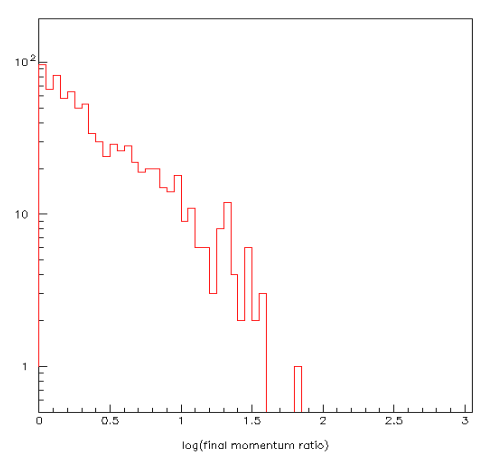

(c)

Fig. 5. Distribution of $\log _{10}\left(E / E_{0}\right)$ below (a), on (b) and above (c) the $H_{0}-M_{0}$ resonance region. From left to right, $H_{0}=1080,3950,0, M_{0}=27,790,1000$ and $\rho=0.27,0.41,0.42$ respectively. The resonance case (central panel) is fit to a power law with index $\sim$ unity.

give evidence for the necessity of bouncing the particle being accelerated on magnetic turbulences rather than bending its trajectory in a uniform magnetic field. A uniform magnetic field can only double the particle energy while magnetic turbulences, here modelled as a lattice of magnetic dipoles oriented at random but having a common magnetic moment, are able to increase the particle energy by several orders of magnitude.

For the acceleration to be efficient, the contribution of the turbulences to the total magnetic field must be commensurate with that of a possible uniform component that may subsist from the parent Supernova. Moreover the mesh size of the lattice, namely the distance between neighbour turbulences, must be significantly larger, by at least an order of magnitude, than the Larmor radius of the cosmic ray. When acceleration proceeds, the Larmor radius increases, and the latter condition causes the cosmic ray to ultimately escape downstream. However, the condition is sufficiently loose to typically allow for acceleration by three orders of magnitude.

Energy spectra are found to obey a power law with index close to unity when the conditions of acceleration are satisfied. When they are not, the main effect is the appearance of a cut-off associated with the early escape of the cosmic ray downstream. The standard DSA calculation predicts a value of 2 for the index under the hypothesis of isotropy of the momenta and of an escape probability proportional to energy. The conditions realised in the present simulation are indeed more favourable.

It is important at this stage to underline the numerous approximations that have been made and the many differences that exist between model and reality. We list below the most relevant:

- The front is assumed to be planar but it is rather spherical. Allowing for enough time, the particle that drifts downstream will ultimately meet again the shock inside which it is enclosed. In the case of a young Supernova, a few light years may be sufficient. 
- The problem of injection has been ignored, the cosmic ray was assumed to have already reached energy of $10 \mathrm{GeV}$ before starting the acceleration cycle. The parameter of relevance is the ratio between the Larmor radius and the thickness of the front, which must be large enough for the front to be seen as a discontinuity. It seems now established [10] that the tail of the thermal Maxwell-Boltzmann distribution is in practice sufficient to feed injection at the desired rate.

- An essential feature is the interaction between the cosmic ray and the interstellar medium, producing streaming instabilities. This mechanism was completely ignored in the present work and a realistic simulation of the plasma waves induced by the accelerated cosmic rays is a very difficult problem. The energies involved in the magnetic turbulences are commensurate with that of the cosmic rays and it is therefore very reasonable to assume that important interactions would result. It is also reasonable to assume that the scale that characterizes such turbulences is commensurate with the Larmor radius of the cosmic rays. But to say anything beyond these qualitative remarks is far from obvious and far beyond the scope of the present article.

- The choice of a square lattice of randomly oriented dipoles, all having the same magnetic moment is certainly a sensible choice: it automatically satisfies Maxwell equations and gives a fair qualitative representation of turbulences. However, quantitatively, it is very far from reality and is a very crude picture of what is going on. In particular, in the case of small Larmor radii, it is too idealized to properly describe magnetic trapping: it reveals its possible importance, but cannot claim to achieve more than that. Recent studies of scale interactions in magneto-hydrodynamic turbulence [11] have shed light of possible turbulence fluctuations and illustrated the complexity of the problem, well beyond the scope of the present work.

- In reality, both the uniform magnetic field component and the magnetic turbulences have average amplitudes that depend on the distance to the shock front and, more importantly, are different upstream and downstream. This effect is ignored in the present model.

- Finally a compression ratio of 4 , typical of hydrodynamic shocks, was assumed without justification.

It is clear from this list that the model used in the present simulation must limit its ambition to a qualitative illustration of the mechanism of diffusive shock acceleration. Its value is only didactic. Yet, diffusive shock acceleration is such a complex process, difficult to visualize, that having a simple picture of it helps with a deeper understanding of its mechanism.

More realistic models must be made in the framework of plasma physics. However, even the most sophisticated of these cannot hope for an accurate quantitative description of the real situation, the complexity of which is well beyond present modelling abilities.

\section{ACKNOWLEDGEMENTS}

We thank Dr. Pierre Lesaffre for a careful reading of the manuscript and important suggestions for improvement. We acknowledge financial support from the Vietnamese Ministry of Science and Technology and from the World Laboratory. 


\section{REFERENCES}

[1] J. Abraham et al. (Auger Collaboration), Science 318 (2007) 938.

[2] J. A. Hinton (HESS Collaboration), New Astronomy Review 48 (2004) 331.

[3] H. Muraishi et al. (CANGAROO Collaboration), Astron. and Astrophys. 354 (2000) L57; R. Enomoto et al. (CANGAROO Collaboration), Nature 416 (2002) 823.

[4] F. Aharonian et al. (HESS Collaboration) Nature 432 (2004) 75.

[5] E. Pfefermann and B. Aschenbach, ROSAT observation of a new SNR in constellation Scorpius, Röntgenstrahlung from the Universe, 1996, 267.

[6] D. C. Ellison, A. Decourchelle and J. Ballet, Astron. and Astrophys. 413 (2004) 189.

[7] Y. Uchiyama et al., Nature 449 (2007) 576 and references therein.

[8] R. Blandford and D. Eichler, Phys. Rep. 154 (1987) 1; S. P. Reynolds, Astrophys. J. 493 (1998) 375; Y. Jiang et al., arXiv : 0904.4918 (2009); E.G. Berezhko and D. C. Ellison, Astrophys. J. 526 (1999) 385; E.G. Berezhko and H.J. Völk, Astron. and Astrophys. 451 (2006) 981; D.C. Ellison et al., Astrophys. J. 661 (2007) 879; A. E. Vladimirov, Astrophys. J. 688 (2008) 1084.

[9] H.J. Völk, E.G. Berezhko and L.T. Ksenofontov, Proc. 29 ${ }^{\text {th }}$ Int. Cosm. Ray Conf., Pune, 3 (2005) 233.

[10] E. Parizot, Accélération des Particules: les Mécanismes de Fermi, Physique et Astrophysique du Rayonnement Cosmique, Ecole CNRS de Goutelas XXVI (2003), p. 124.

[11] For a recent review see P.D. Mininni, Scale Interactions in Magnetohydrodynamic Turbulence, arXiv:1006. 1817v1 [physics.flu-dyn] 9 Jun 2010, and references therein.

Received 29 November 2010. 